\title{
Diamonds for MRI
}

Three groups report key steps toward nanometer-scale magnetic resonance imaging using nitrogen-vacancy centers in diamond.

A tiny defect in diamond, called the nitrogen-vacancy (NV) center, is currently demonstrating big potential for applications cutting across scientific fields. Among their many potential uses, these NV centers can serve as atom-sized magnetic field sensors. This unique property is generating much excitement about the potential for magnetic resonance imaging (MRI) on the nanometer scale.

Recent work published in Nature Nanotechnology from Daniel Rugar of IBM Research, David Awschalom of the University of Chicago and colleagues (Rugar et al., 2015), Friedemann Reinhard and Joerg Wrachtrup of the University of Stuttgart (Häberle et al., 2015), and Ronald Walsworth and his team at Harvard University (DeVience et al., 2015) reports key development steps to this NV center technology, which hit the ground running in 2008. "These papers report the next steps in a larger story about the rapid growth of the field of atom-like quantum sensors in diamond that are beginning to have a major impact in a lot of different fields," says Walsworth.

The Rugar-Awschalom team showed that a single NV center just below the diamond surface sensed an oscillating magnetic field from protons in an organic polymer sample attached to a tuning fork as it was scanned past the NV center. From this, they generated a two-dimensional image of the polymer sample with a spatial resolution of 12 nanometers. The Reinhard-Wrachtrup group attached their sample (a sphere of engraved Teflon, a fluorocarbon polymer) to an atomic force microscopy cantilever and scanned it over a diamond sensor containing a single NV center, producing an image of the engraved nanostructure with 10-nanometer resolution. And the Walsworth team demonstrated detection of multiple nuclear species- ${ }^{1} \mathrm{H},{ }^{19} \mathrm{~F}$ and ${ }^{31} \mathrm{P}$-in various smallmolecule samples. Like the other groups, they performed a scanning-type experiment with a single NV center, but they also demonstrated the potential for wide-field MRI, using a dense, shallow layer of many NV sensors just below the diamond surface.
Although these studies represent important steps in advancing the technology, Walsworth stresses their proof-of-principle nature. "We want to continue to make the technology work better-higher resolution, better sensitivity, faster-but increasingly apply it to interesting samples," he says. The potential is great: the combination of magnetic field sensitivity and spatial resolution is currently unmatched by any other technology. And the diamond sensors operate under ambient conditions, at room temperature, which in principle should pose no limitations on biological applications.

Among such applications, the possibility of performing nanoscale-resolution MRI will enable nondestructive imaging at a substantially smaller scale than that of traditional, micrometer-scale, MRI. It remains to be studied whether there is sufficient MRI contrast to resolve intracellular structures, Walsworth notes.

One of the most intriguing applications is the potential to perform single-protein structure determination, perhaps even in situ. However, applying this nascent technology to do real structural biology will be no easy task, says Walsworth. "We're going to need to register the molecules over the NVs and make sure that the molecular motion relative to the NVs does not obscure the atomic-scale magnetic signal," he notes. "We've got to be able to pull out information about the couplings that are going on between the atoms in those molecules." He suggests that previous structural information could be used to design smart pulse sequences intended to target particular spots on biomolecules where structure is unknown. Techniques adapted from the quantum-information community may also prove to be useful.

It will certainly be exciting to watch the progress in this field and see whether these defective diamonds will sparkle brightly as research tools.

Allison Doerr

\section{RESEARCH PAPERS}

DeVience, S.J. et al. Nanoscale NMR spectroscopy and imaging of multiple nuclear species. Nat. Nanotechnol. 10, 129-134 (2015).

Häberle, T. et al. Nanoscale nuclear magnetic imaging with chemical contrast. Nat. Nanotechnol. 10, 125-128 (2015).

Rugar, D. et al. Proton magnetic resonance imaging using a nitrogen-vacancy spin sensor. Nat. Nanotechnol. 10, 120-124 (2015). 